\title{
A Modal Analysis of the Irradiation Instability
}

\author{
Jeffrey Fung ${ }^{1}$ and Pawel Artymowicz ${ }^{1,2}$ \\ ${ }^{1}$ Department of Astronomy and Astrophysics, University of Toronto, \\ 50 St. George Street, Toronto, Ontario, Canada M5S3H4 \\ email: fung@astro.utoronto.ca \\ ${ }^{2}$ Department of Physical and Environmental Sciences, University of Toronto at Scarborough, \\ 265 Military Trail, Scarborough, Ontario, Canada M1C1A4 \\ email: pawel@utsc.utoronto.ca
}

\begin{abstract}
The irradiation instability is a disk instability caused by the radiation pressure cast by a central source onto an optically thick disk. The criterion for this instability depends on a sharp transition from an optically thin inner disk to an optically thick outer disk. The quickly diminishing radiation pressure in this transition region creates a radially compressing effect, which is in many ways similar to the effects of self-gravity. In this modal analysis, we demonstrate that a disk marginally stable to irradiation can develop global modes, with growth rates being of order the dynamical timescale of the disk. The non-linear evolution of the our model shows the formation of vortices near the transition region and spiral structures propagating into the optically thick region. Consequently the scale-height of our disk's inner edge becomes timevariable and can likely be observed as a variation in its infrared flux.
\end{abstract}

Keywords. instabilities, hydrodynamics, scattering, planetary systems: protoplanetary disks, accretion disks

Instabilities in accretion disks provide a mechanism for transferring angular momentum outward, resulting in accretion flows. Those that have been considered important to the evolution of accretion disks include the magnetorotational instability (MRI) (Balbus \& Hawley 1998), gravitational instability (Lin \& Pringle 1987, Gammie 2001), PapaloizouPringle instability (Papaloizou \& Pringle 1984, 1985, 1987), and Rossby wave instability (RWI) (Lovelace et al. 1999). The list goes on if non-ideal MHD and the vertical shearing (Urpin \& Brandenburg 1998) are also considered. Here we introduce a new form of instability associated with the radiation pressure exerted by a central object onto an optically thick disk which contains an inner edge. We term this instability the "irradiation instability".

The common parameter for describing the force of radiation pressure is $\beta_{0}$ :

$$
\beta_{0}=\frac{\kappa_{\mathrm{opa}} L_{*}}{4 \pi c G M_{*}},
$$

where $L_{*}$ is the host's luminosity, $M_{*}$ is the host's mass, and $\kappa_{\text {opa }}$ is the opacity of the disk material; $G$ and $c$ are the gravitational constant and speed of light respectively. This parameter describes the fraction of gravity cancelled by radiation pressure, as both of them are central forces that diminish as $r^{-2}$. The force due to radiation pressure on dust grains is comparable to gravity, if the source is sufficiently luminous, or the grains are of an optimal size. For example, micron-sized grains under the illumination of an A type star have a $\beta_{0}$ well exceeding unity.

Radiation pressure can potentially destabilize disks. Consider the following: submerged in a disk where a porton of the illumination from the central source is blocked by layers of 


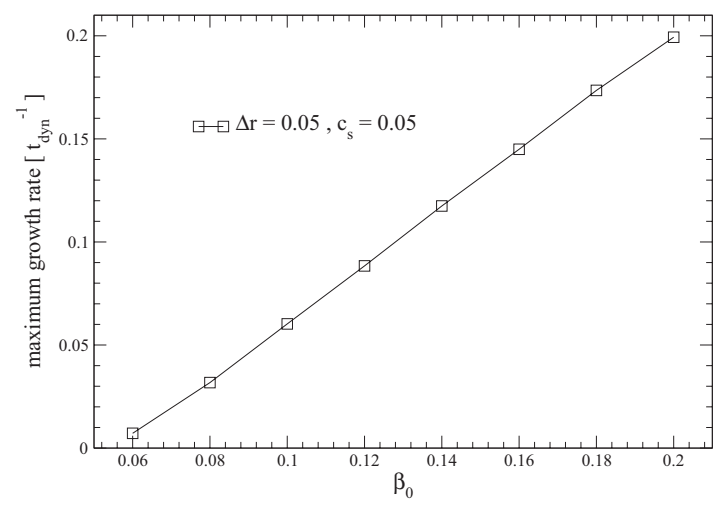

Figure 1. The growth rate of the asymmetric mode increases with $\beta_{0}$. There is no measurable modal growth for $\beta_{0}<0.06$. $\Delta r$ and the sound speed $c_{\mathrm{s}}$ are non-dimensionalized, such that $r=1$ and the Keplerian velocity $v_{\mathrm{k}}=1$ at the inner edge of the disk.

dust, an over-dense dust cloud slowly passes in front of a dust grain. As a result, a darker shadow is cast on to the grain. Consequently, the grain feels less push from radiation pressure, and it migrates towards the over-dense cloud, further increasing the density of the cloud. This process can potentially runaway and cause an instability if the shear in the disk fails to tear these clouds apart in time.

To capture this process, we derive a condition for local stability:

$$
Q_{\mathrm{rad}} \equiv\left(\beta_{0} e^{-\tau} \frac{\Omega^{2}}{\kappa^{2}} \frac{\mathrm{d} \tau}{\mathrm{d} \ln r}\right)^{-1}>1,
$$

where $\tau$ is the radial optical depth, $\kappa$ is the epicyclic frequency, and $\Omega$ is the orbital frequency.

We perform a modal analysis where we solve the linearized set of Euler's equations and calculate the growth rates for individual azimuthal modes. We use a density profile that is constant, but with an inner edge where the density gradually falls to $1 \%$ of its value over a distance $\Delta r$. The $\tau$ profile is normalized such that $\tau=2 / 3$ at the inner edge. Using this disk model, fig. 1 shows that the instability can grow as fast as a few dynamical timescales. While the growth rates plotted in fig. 1 do depend on our disk model, it is important to note that the disk flux is stable in the limit of a small $\beta_{0}$ demonstrating that radiation pressure is the driving force that destabilizes our disk.

We also investigate the fully non-linear regime of this instability through numerical simulations. We find that the asymmetric modes develop into vortices that remain at the inner edge of the disk. These features create a variable scale height at the inner edge and can result in an observable infrared variability (Flaherty \& Muzerolle 2010).

\section{References}

Balbus, S. A. \& Hawley, J. F. 1998, Reviews of Modern Physics, 70, 1

Flaherty, K. M. \& Muzerolle, J. 2010, ApJ, 719, 1733

Gammie, C. F. 2001, ApJ, 553, 174

Lin, D. N. C. \& Pringle, J. E. 1987, MNRAS, 225, 607

Lovelace, R. V. E., Li, H., Colgate, S. A., \& Nelson, A. F. 1999, ApJ, 513, 805

Papaloizou, J. C. B. \& Pringle, J. E. 1984, MNRAS, 208, 721

Papaloizou, J. C. B. \& Pringle, J. E. 1985, MNRAS, 213, 799

Papaloizou, J. C. B. \& Pringle, J. E. 1987, MNRAS, 225, 267

Urpin, V. \& Brandenburg, A. 1998, MNRAS, 294, 399 